Johnson \& Johnson

\section{Raucherentwöhnung in der zahnärztlichen Praxis nachhaltig unterstützen}

Im Rahmen der Fortbildungsveranstaltung „practica“ hat die Johnson \& Johnson GmbH ein gemeinsam mit Ärzten und Zahnärzten entwickeltes Programm zur Raucherentwöhnung in der Praxis vorgestellt. EINFACH ERFOLGREICH RAUCHFREI bündelt wissenschaftliche Informationen und praktische Tipps zur Raucherentwöhnung. Evidenzbasiert gibt das Modell präzise Handlungsempfehlungen mit dem Ziel, Raucherentwöhnung einfach und erfolgsorientiert zu gestalten. Zur Anwendung in der zahnärztlichen Praxis kommt die Nicotinsubstitution beispielsweise in Form des NICORETTE ${ }^{\circledR}$ TX Nicotinpflasters.

Dr. med. dent. Holger Gehrig, Kandel: „Es ist viel einfacher, als man denkt, Patienten anzusprechen und anzuleiten. Unser Leitfaden zur Gesprächsführung gibt Ihnen Tipps und Hilfestellungen, wie Sie geeignete
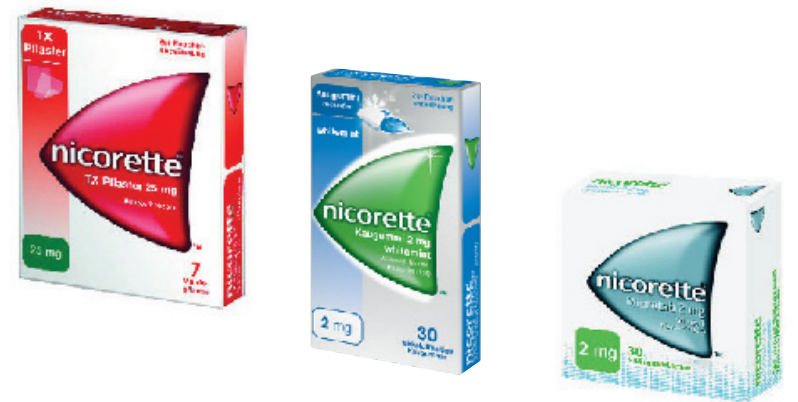

Johnson \& Johnson

Patienten identifizieren und wie Sie die Termine und Konversationen mit Rauchern gestalten können." Mit einem vergleichsweise geringen Aufwand kann die Raucherentwöhnung somit einen gesundheitlichen Nutzen erbringen. Alle Dokumente können kostenlos auf www.einfach-erfolgreich-rauchfrei.de angefordert werden.

$\pi$ www.nicorette.de

\title{
Carestream Health
}

\section{Frank Berlinghoff übernimmt Marketing-Leitung}

Frank Berlinghoff hat bei Carestream Dental die Marketing Leitung Kodak Dental Systems für die Regionen Europe, Africa und Middle East (EAMER) übernommen.

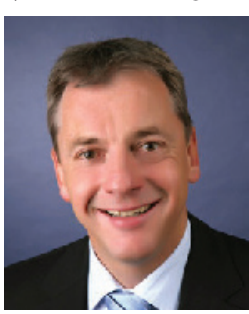

Berlinghoff verfügt über mehr als 20 Jahre Dentalerfahrung in leitenden Positionen nationaler und internationaler Dentalunternehmen. Der studierte Physik-Ingenieur war u.a. in verschiedenen Tätigkeiten bei KaVo beschäftigt und unterhält langjährige Kontakte zu den deutschen Dental-Universitäten. Gemeinsam mit Dave Pullen (General Manager EAMER), Nicola Gizzi (Sales \& Service Director) und Frank Bartsch (Trade Marketing
Manager) wird Berlinghoff seine Kompetenzen dafür nutzen, Carestream Dental mit seinen vielseitigen Angeboten rund um bildgebende Verfahren auch in Zukunft erfolgreich am Markt zu positionieren. „Ich freue mich, meine Dentalerfahrungen bei Carestream Dental einzubringen und den Zahnärzten in Sachen Diagnose mit unseren Produkten eine bestmögliche Auflösung zu ermöglichen", so Berlinghoff selbst.

\section{$\boldsymbol{\lambda}$ Tel. 0711 - 20707-306}

europedental@cshdental.com

www.kodakdental.com

lege artis

\section{Vorsprung auf einen Blick}

Im deutschen Prophylaxemarkt hat durimplant (Hersteller: lege artis Pharma) für die Implantaterhaltung und zur Zahnfleischpflege eine weite Verbreitung in den Praxen gefunden. Empfohlen wird das Produkt i.d.R. von der Prophylaxe-Mitarbeiterin für Patienten mit Implantaten zur Prophylaxe zuhause, zur Vermeidung von Periimplantitis und Zahnfleischentzündungen bakteriellen Ursprungs. Der Verkauf erfolgt rezeptfrei über die Apotheke oder über den Praxis-Prophylaxeshop.

Zur optimalen Prophylaxe verwendet der Patient durimplant einmal wöchentlich bis einmal täglich abends nach dem letzten Zähneputzen. Hier vorab die wichtigsten Nutzen für den Verwender: durimplant ist als Dauerprophylaktikum zur täglichen Pflege geeignet. Es schmeckt angenehm medizinisch nach Salbei und frisch nach Minze, verbleibt lange vor Ort, wirkt über die ganze Nacht (slow release) und ist leicht und gut zu platzieren.
Weitere Vorteile:

Ideale Kombination pflegender und natürlicher Inhaltsstoffe, Einwirkung mehrstündig über Nacht, angenehmer Geschmack, auch in der Apotheke erhältlich (PZN - 4999590).

Eine übersichtliche Gegenüberstellung der Produkteigenschaften von durimplantzu, normalen' $\mathrm{CHX}$-Gelen finden Sie unter www.durimplant.com .

入 Tel. $07157-56450$

info@legeartis.de www.durimplant.com 